\title{
A Simulation Study on the Schooling Mechanism in Fish*1
}

\author{
Ichiro AoKI ${ }^{* 2}$ \\ (Received December 25, 1981)
}

\begin{abstract}
A computer simulation was performed to examine the schooling mechanism. The model uses the basic assumptions that the speed and direction of individual movements are stochastic variables, and that the direction of movement is related to the location and heading of the neighbors. Three behavioral interactions between individuals were considered; approach, avoidance, and parallel orientation movements. Individual movements and the resultant group movement were simulated by repeatedly generating random numbers.

It was found that group movements in unity could occur despite each individual lacking knowledge of movement of the entire school, and in the absence of a consistent leader. The results lead to the following conclusions: for effective schooling to occur, individuals must possess two types of behavior; 1 , approach movement to allow aggregation, and 2. parallel orientation movement to allow the group to move cohesively. Experiments were also performed with varied combinations of model parameters, and a wide variation in the movement pattern of the simulated school was obtained.
\end{abstract}

Schooling results from interactions in which an individual control its movement in relation to neighbors and coincidently has an influence on these neighbors. We must answer the question of how individuals interact with one another or what interactions are required to permit schooling. Behavioral properties with which schooling species are endowed have been explored through several approaches, particularly descriptions of behaviroal changes by limiting a sensory clue, ${ }^{1)}$ reactions to stationary or moving objects, ${ }^{2-\delta)}$ and analyses of individual movements within the school..$^{8,7)}$ Consequently, many suggestions have been made to explain the schooling mechanism. Most investigators take an interest in mutual attraction and attribute schooling to this tendency. SHAW ${ }^{8)}$ has suggested two causal factors, mutual attraction and parallel orientation, which may be subject to different neural mechanisms, and emphasized the singificance of the distinction between polarized and nonpolarized forms shown by groups which were based on mutual attraction.

In this paper, by synthesizing these suggestions, computer simulations were performed to examine the schooling mechanism. A model was made to explore the feasibility of cohesive group movement. The simulation approach has the advantages that it is easy to add, exclude, or change a decision rule concerning individual movements.
Therefore, it is possible to evaluate quantitatively the effects of interactions underlying schooling.

\section{The Model}

A numerical stochastic model was developed to simulate the schooling phenomenon. In this approach, the behavior of a system is examined by a procedure in which a basic process having a stochastic aspect is generated and then run on a computer.

The following basic assumptions are made.

1) Time is quantized, and movement is decided at intervals of $\Delta T$. Decisions are independent of the previous step.

2) A hypothetical organism moves in twodimensions on the horizontal plane.

3) The two components of movement, speed and direction, are stochastic variables characterized by probability distributions. Speed and direction are mutually independent.

4) To simplify the problem, interactions between individuals are restricted to the directional component, which is considered more important. ${ }^{9)}$ Thus, the velocity component at any time is determined independently of other individuals.

5) In the initial state, individuals are distributed at random within a square area with sides of a certain length $(I L)$, and their directions of

*1 Experimental and Theoretical Studies on Schooling in Fish-I.

*2 Ocean Research Institute, University of Tokyo, Nakano, Tokyo 164, Japan （青末一郎：東京大学海洋 研究所). 
orientation are given with uniform distribution over the range $0^{\circ}$ to $360^{\circ}$.

The speed and direction of movement are determined for each individual according to the following decision rules.

The velocity distribution is described by a gamma distribution, namely, the probability density is given by

$$
f(v)=\frac{A^{K}}{\Gamma^{\prime}(K)} e^{-A v} v^{K-1}
$$

where $v$ is velocity, $K$ and $A$ parameters, $v \geq 0$, $K>0, A>0, \Gamma(K)$ is gamma function, and if $K$ is an integer, $\Gamma(K)=(K-1)$ !. The values of the constituent parameters $K$ and $A$ are constant, and based on previous observations. ${ }^{\text {p) }}$ The distance moved is expressed in body lengths (BL).

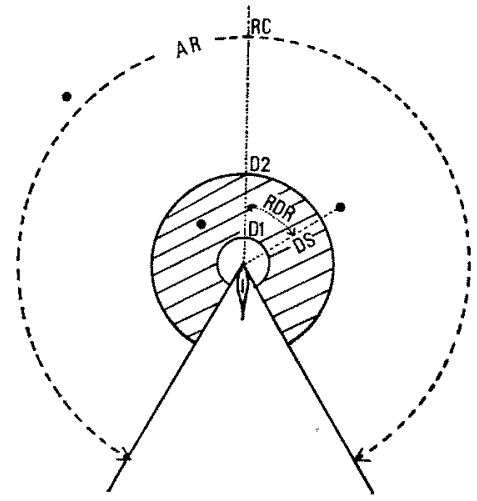

Fig. 1. Geometrical drawing to illustrate parameters specifying interactions.

$D 1$, avoidance distance; $D 2$, approach distance; $R C$, radius of extent of near-field interactions; $A R$, angular range of interactions; shadowed portion, parallel orientation area; solid circles locations of other individuals (distance denoted by $D S$ and relative direction by $R D R$ ).

To determine the directions of movement (heading) a normal distribution was chosen with one exception described below. The mean and variance of the heading are defined in relation to the location and heading of neighbors in a sector area defined by the angle $A R$ and the radius $R C$ (See Fig. 1). The probability density for the direction of movement of the $i$-th individual $p_{i}(\theta)$ is given by

$$
p_{i}(\theta)=\sum_{j} W_{j} \frac{1}{S_{j} \sqrt{ } 2 \pi} e^{-\left(g-B I j^{2}\right) / 2 S^{\prime}}
$$

where $W_{f}$ is a weighting factor to scale the in- fluence of the $j(\leq 4)$ neighbors within the distance $R C$ and closest to the directional heading of $i$-th. The numerical values for the relative weights $W_{1}$ were defined as inversely proportional to the relative magnitude of the angle $R D R\left(0^{\circ} \leq R D R \leq\right.$ $180^{\circ}$ ), the angle between the heading of the $i$-th individual and the location of its $j$-th neighbor. The relative influence of these neighbors was defined as

$$
W_{j+1}=R F \cdot W_{j}
$$

and

$$
\sum_{j} W_{j}=1, j=1, \cdots, j_{m}\left(j_{m} \leq 4\right),
$$

where $R F$ is a constant $(0 \leq R F \leq 1)$ set prior to each simulation. Defining an avoidance distance $D 1$, an approach distance $D 2$, the direction of the $j$-th neighbor as $D R_{j}$ and the heading of the $j$-th neighbor as $D H_{j}$, then $M_{j}$ and $S_{j}$ are determined as follows,

1) when $D S_{f}>D 2, M_{f}=D R_{f}$ and $S_{f}=S D 1$ (constant), which allows approach,

2) when $D 1 \leq D S_{j} \leq D 2, M_{j}=D H_{j}$ and $S_{j}=$ $S D 2$ (constant), which allows parallel orientation, and

3) when $D S_{j}<D 1, M_{j}$ is chosen as either $D R_{j}+90$ or $D R_{j}-90$, depending upon which heading forms the smaller angle with the heading of individual $i$.

The boundary and the weighting factor for influence are introduced on the basis of several facts, the character of swimming forward, the extent of the visual field, the occulation of the sensory path by other fish, and the tendency that a neighbor ahead of a given fish is used more frequently as an angular reference than neighbors to the side. ${ }^{\text {) }}$

If, for a given individual, neighbors are not found in the sector area defined above but some are in the range of the angle $A R$ regardless of the distance, the approach motion toward a nearer neighbor is set up. Namely, $M_{j}$ and $S_{j}$ are determined similarly to the above case 1 ), but the weighting factor $W$, based on the increasing magniture of the distance separating the $j$-th from the $i$-th individual.

Furthermore, if no others are found in the range $A R$, the direction of movement is determined with uniform probability over the range $0^{\circ}$ to $360^{\circ}$ independently of others.

The model described above was programmed to perform the necessary judgements and calculations, and run practically following the procedure shown by Fig. 2 . 


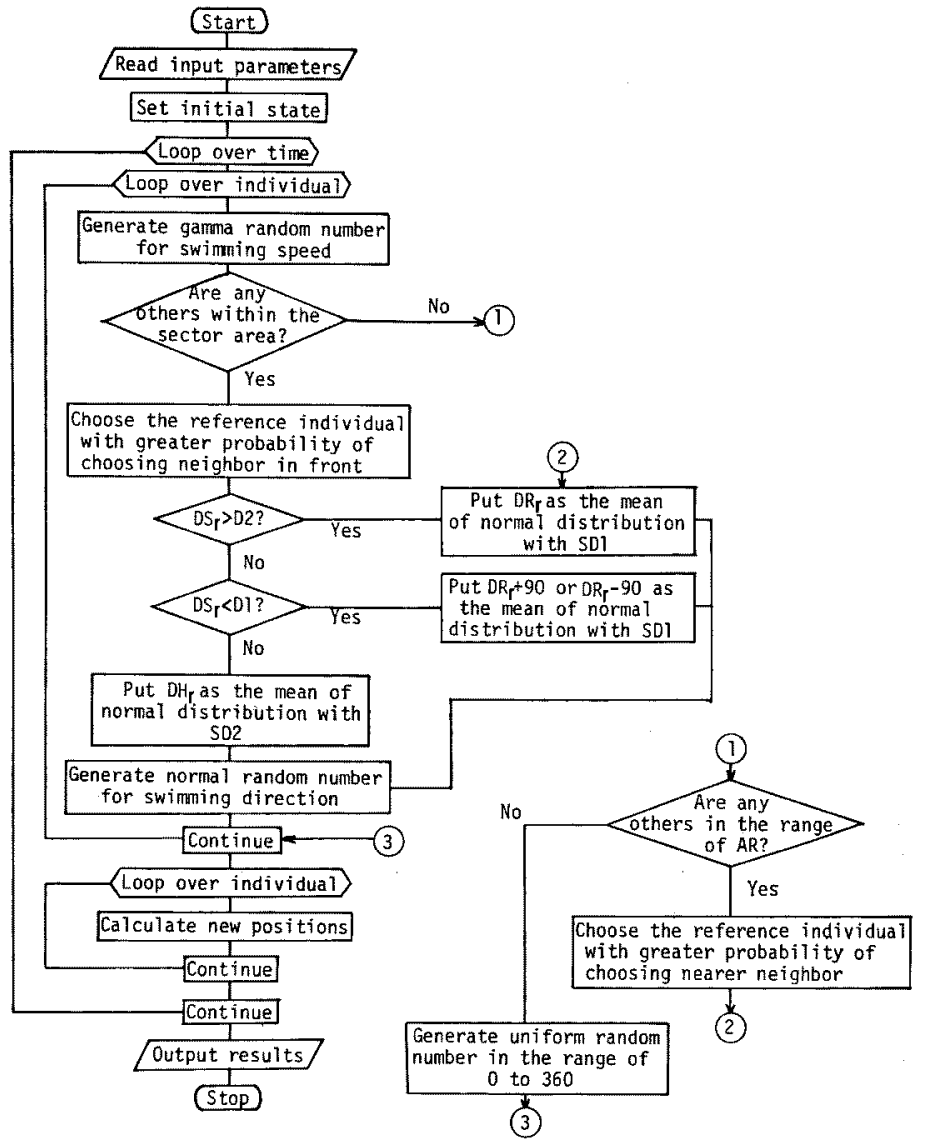

Fig. 2. Flow_chart of the simulation model.

$D S_{r}$, distance of the reference individual from a given individual; $D R_{r}$, direction of the reference individual from a given individual; $D H_{r}$, heading of the reference individual; $A R$, angular range of interactions; $D 1$, avoidance distance; $D 2$, approach distance; $S D 1$, standard deviation for the direction of avoidance and approach movements; $S D 2$, that for the direction of parallel orientation movement.

\section{Results}

\section{The Standard Run}

Parameters in the model specify the feature of the movement pattern of a group. Since we could not afford to run all possible combinations of a wide variety of values, a standard simulation was run with a basic set of values, against which modification runs were compared to examine the influence of changing in the parameters.

Table 1 shows the values of the parameters used in the standard run. The choice of these values was made so as to be consistent with available data on the distance and angular deviation between fish in schools, ${ }^{8, \theta)}$ though the value of $R F$ was arbitrary. The average density at the initial state corresponded to one individual per circle area of radius $0.92 \mathrm{BL}$. The mean and standard deviation of the speed were $1.21 \mathrm{BL} / \mathrm{sec}$ and $0.606 \mathrm{BL} /$ $\mathrm{sec}$, respectively. The number of individuals was relatively small, eight for simplicity and economy of computing time.

For each set of parameters, a simulation run was continued through 2000 steps, and further the entire process was repeated twenty times. Simulations were carried out using the computer FACOM-M160 and the results output on a lineprinter and a X-Y plotter. A micro-computer (APPLE II) was sometimes employed and the movement pattern displayed on a TV screen.

Fig. 3 shows an example of results for the standard run. The paths for two individuals 
Table 1. Values of parameters used in the standard run

\begin{tabular}{llc}
\hline \multicolumn{1}{c}{ Parameters } & Abbreviations & Values \\
\hline Number of individuals & $N$ & 8 \\
Final step length & $T M A X$ & 2000 \\
Time interval of step & $\Delta T$ & $0.5(\mathrm{sec})$ \\
Range of initial locations & $I L$ & $4.6\left(\mathrm{BL}^{*}\right)$ \\
Parameters of gamma distribution & $K$ & 4 \\
& $A$ & 3.3 \\
Standard deviations of normal distribution & & \\
$\quad$ Approach and avoidance & $S D 1$ & $15(\mathrm{deg})$ \\
Parallel orientation & $S D 2$ & $15(\mathrm{deg})$ \\
Avoidance distance & $D 1$ & $0.5(\mathrm{BL})$ \\
Approach distance & $D 2$ & $2.0(\mathrm{BL})$ \\
Radius of extent of near-field interactions & $R C$ & $5(\mathrm{BL})$ \\
Angular range of interactions & $A R$ & $150(\mathrm{deg})$ \\
Reference factor in interactions & $R F$ & 0.5 \\
\hline
\end{tabular}

- Body length (BL) was used as the unit of distance.
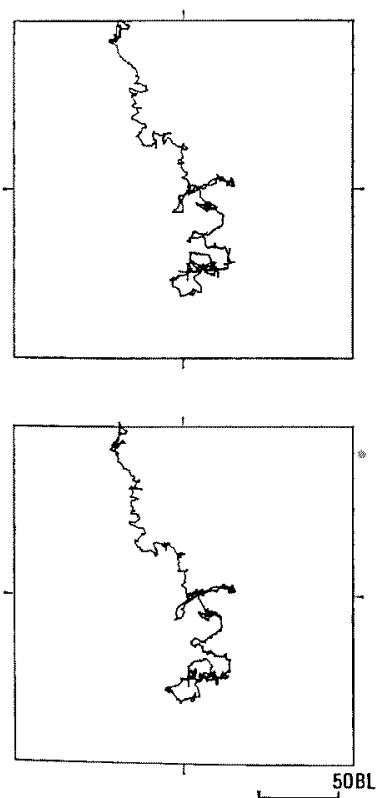

(1)
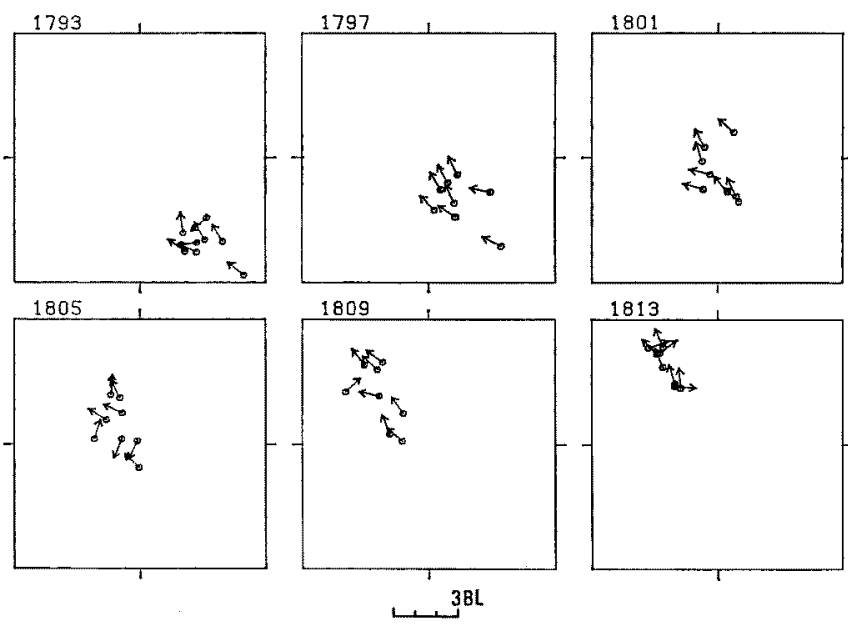

Fig. 3. Examples of movement patterns of the simulated school in a standard run.

(1) Examples of paths taken by individuals, (2) locations (circles) and headings (arrows) of individuals.

show fair agreement as expected. The detailed movement patterns of the simulated school are also shown by plotting the position and the direction of movement for each individual. We found that group movement in unity occurred despite each individual lacking knowledge of movement of the entire school, and in the absence of a consistent leader.

A measurement of the spread of the simulated school was made by calculating the standard deviations of the $x$ and $y$ coordinates at each step (Fig. 4). Though exhibiting occasional increases to over $4 \mathrm{BL}$, which were followed by rapid declines to around $1 \mathrm{BL}$ the standard deviations were comparatively stable. In this example, time averaged values of the standard deviations were 1.11 BL for the $x$-axis and $1.05 \mathrm{BL}$ for the $y$-axis, which were slightly lower than those obtained for a real eightfish school of Gnathopogon. ${ }^{\text {) }}$ The standard deviation of coordinates showing the spread of a 


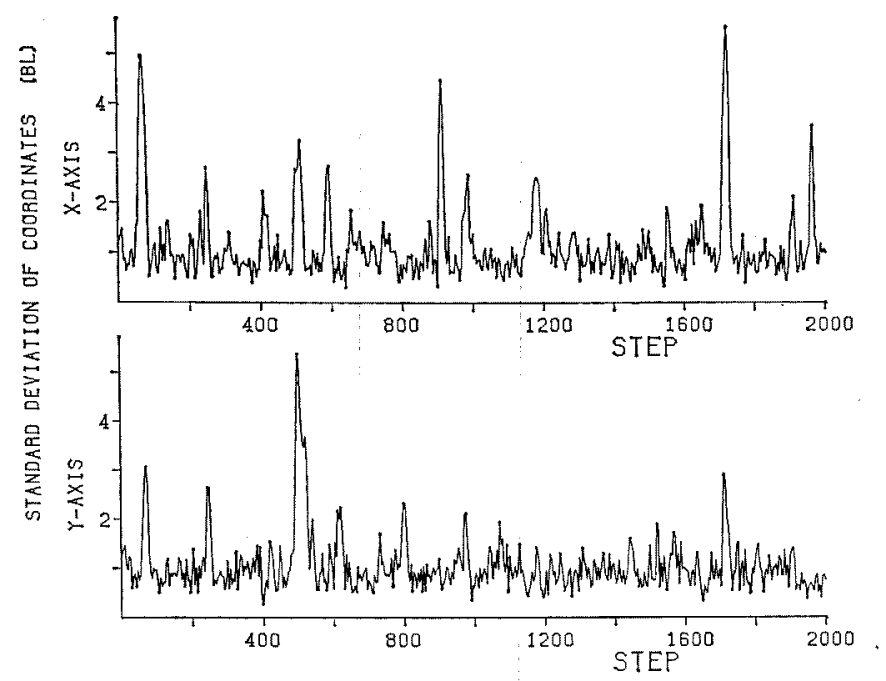

Fig. 4. Time series of the spread of the simulated school in a standard run.

school can also be taken as a measure of compactness so long as the number of individuals in a school is taken into account. Thus, the simulated school is found to show a realistic compactness. The standard deviations of coordinates can also facilitate comparisons of compactness between the standard run and modifications.

\section{Effects of Changes in Parameters and Decision Rules}

Experiments with the values of the parameters and the decision rules in the model varied provide insight into the mechanism of schooling and the wide variation in appearance of schools. For each modification, only the value of a parameter or a part of the decision rules was altered while the remaining parameters and the decision rules were not varied.

First, to further examine the reality, the model was run with the number of individuals increased by four times compared with the standard run $(N=32)$. All of the individuals aggregated, moving stably as a group throughout the 2000 steps (Fig. 5). In this example, the time averaged values for the standard deviations of coordinates were $1.85 \mathrm{BL}$ for the $x$-axis and $1.83 \mathrm{BL}$ for the $y$-axis, which were about double the values obtained in the standard run. Thus the spread of the simulated 32-fish school in the two-dimensional plane increased by about four times while the number of individuals also increased by four times. Therefore, it is shown that the 32-fish school was similar in compactness to the 8-fish school in the standard run. These results show

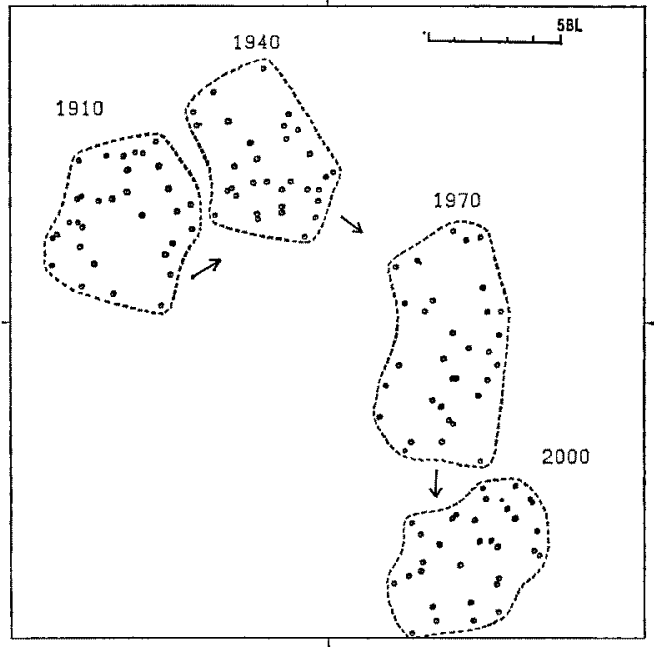

Fig. 5. An example of movement patterns of a simulated school with a larger number of individuals $(N=32)$.

stability of the model.

A second set of simulations made was to examine how movement patterns changed when parallel orientation movement was removed. The same value was chosen for both the approach distance $D 2$ and the avoidance distance $D 1$, which meant that only approach and avoidance movements worked as interactions. Fig. 6(1) shows examples for such individuals. Individuals, though aggregating, tended to wheel, and the range of movement was narrow compared with patterns observed in the standard run. To make 

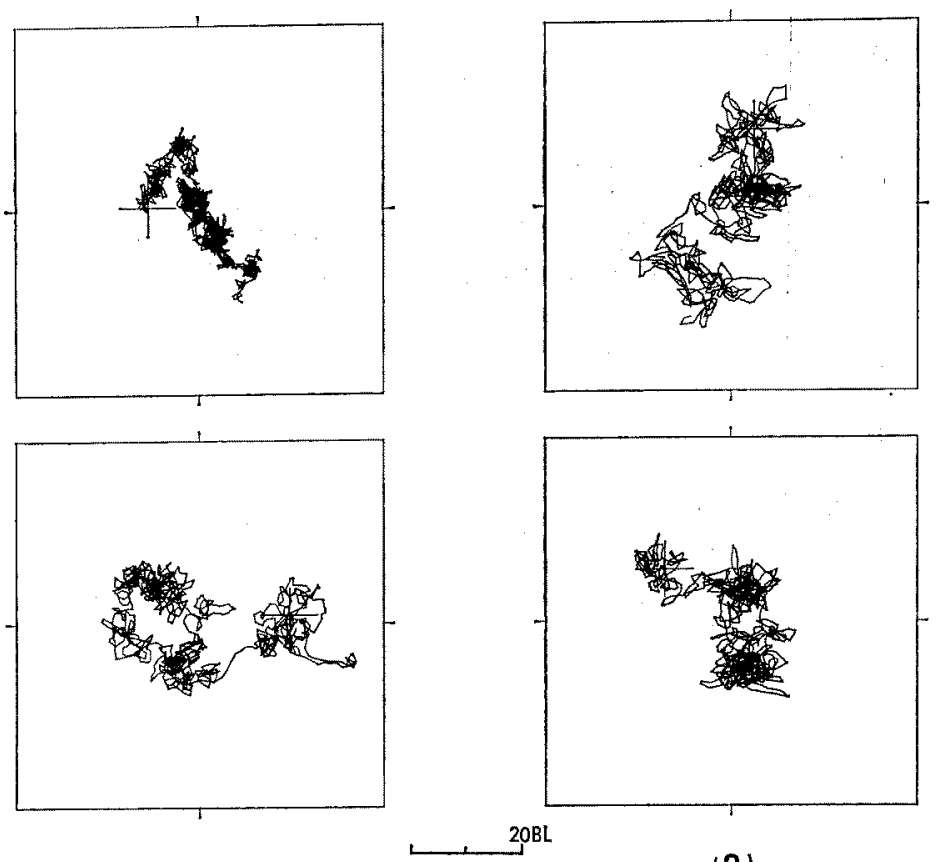

(1)

(2)

Fig. 6. Examples of paths taken by individuals lacking parallel orientation movement.

(1) Only approach and avoidance movement (upper, $D 1=0.5$ and $D 2=0.5$; lower, $D 1=$ 2.0 and $D 2=2.0$ ), (2) forward movement instead of parallel orientation movement (both, $D 1=0.5$ and $D 2=2.0$ ).

this difference more quantitative, the separation between the two most distant positions in the path of individual was used as a measure of mobility. The mean values of the separation for these cases were $1 / 5$ to $1 / 3$ that of the standard run (Fig. 8, details of this figure will be referred to later).

If individuals tend to move ahead in self-dependence when finding a neighbor at a proper distance, is mobility greater despite a lack of parallel orientation movement? The decision rules for the direction of movement was altered partially by replacing parallel orientation movement with forward motion in the region between approach and avoidance distances, namely, when $D 1<D S_{f}<D 2, M_{f}=i$-th individual's own swimming direction at the previous step, and $S_{j}=S D 2$. Fig. 6(2) is an example of paths taken by such individuals, where the values of $D 1$ and $D 2$ were kept the same as in the standard run $(D 1=0.5$ and $D 2=2.0$ ). The types of trajectories were similar to those seen in Fig. 6(1). The behavior of the simulated school was little different in mobility when compared with the previous experiments, though similar in compactness to the standard run (Fig. 8). It is clear that forward movement is not a substitute for parallel orienta-

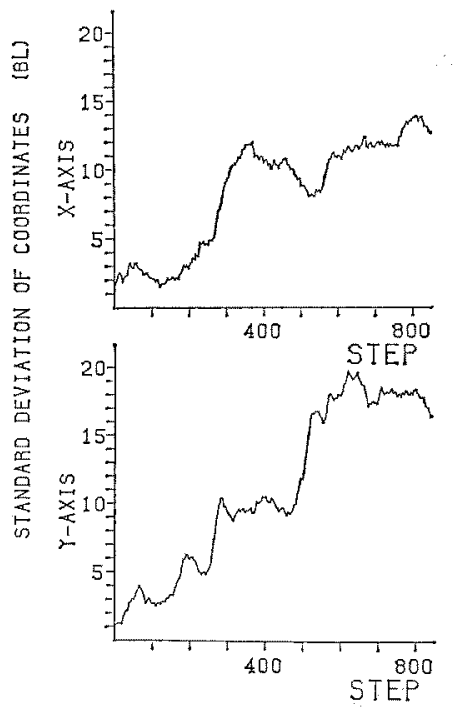

Fig. 7. Time series of the spread of the simulated school in the case when approach and avoidance movement were removed. 


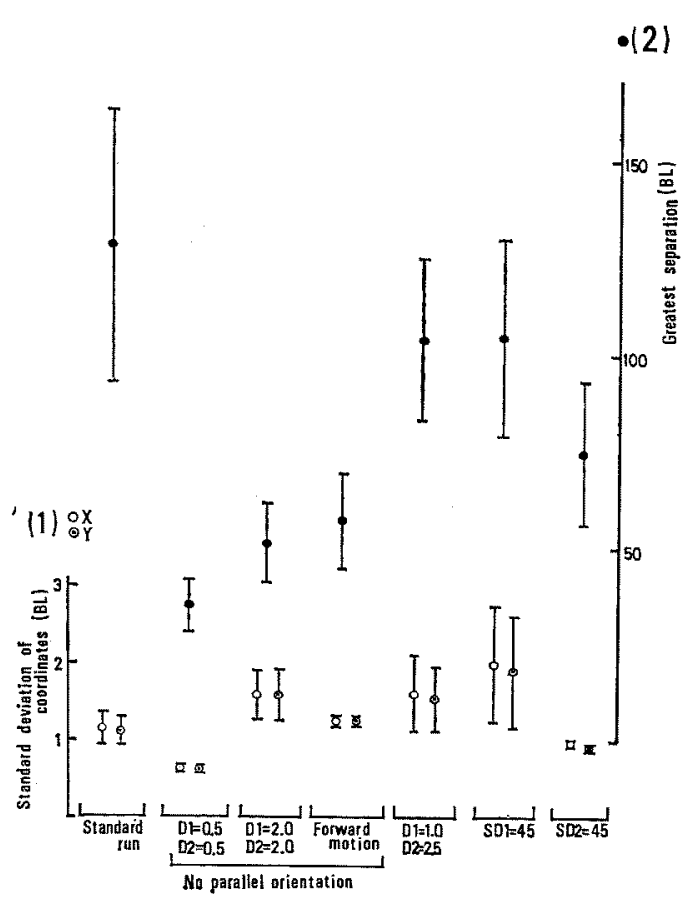

Fig. 8. Effects of parameters and decision rules on compactness and mobility of the simulated school.

Compactness is represented by the reciprocal of the standard deviation of coordinates showing the spread of the simulated school, and mobility by the greatest separation between two points in the path of an individual.

Circles and bars indicate mean and standard deviation for twenty runs, respectively.

See text for full explanation.

tion movement. In contrast with these runs, when approach and avoidance movements were removed and only parallel orientation movement worked ( $D 1=0.0$ and $D 2=1000)$, the individuals dispersed (Fig. 7).

These results lead to the following conclusion: individuals must possess two types of behavior; approach movement to allow aggregation and parallel orientation movement to allow the group to move cohesively.

The next question of interest is how compactness and mobility are affected by values of the parameters $D 1, D 2, S D 1$, and $S D 2$ which determined the interaction boundaries and errors in directivity. The results of these modified runs are summarized in Fig. 8. When the width of parallel orientation area was the same but values of $D 1$ and $D 2$ were $0.5 \mathrm{BL}$ greater in comparison with the standard run, compactness was lower while mobility was not significantly different. Similar results were also obtained when the standard deviation of the direction of approach and avoidance movements was increased to $45^{\circ}$ (SD1 $=$ 45 ), but when the standard deviation of the direction of parallel orientation movement was $45^{\circ}$ $(S D 2=45)$ mobility decreased by one half compared with the standard run although compactness increased only slightly.

The results of the modified simulation runs can be summarized clearly in relation to compactness and mobility of the simulated school. We found that compactness was affected by conditions of approach and avoidance movements and mobility by parallel orientation movement.

\section{Discussion}

The simulation approach allows a better understanding of the behavioral mechanisms involved in fish schooling. Computer simulation may also have applications in suggesting methods for controlling the behavior of fish schools.

SAKAI and SUzUKI ${ }^{10}$ ) have modeled schooling by an equation of motion with an approximation of individual movements by a dynamic process. Also, there have been modifications of that model, a simplified model ${ }^{11)}$ and a developed model introducing fish behavior to a fishing gear. ${ }^{12)}$

The model presented here is based on the assumption that the speed and direction of individual movement are stochastic variables. Therefore, individual movements and the resultant group movement could be simulated by repeatedly generating random numbers which determined the moved distance and direction at each step. Such a simulation model is simple to treat due to its concrete expression. Since some stochastic aspects are found in animal movement, the Monte Carlo method has been widely employed in behavioral and ecologocal research. ${ }^{18-18}$ )

In this model, if an individual find no others in the sector area but some in the angle $A R$, it approaches to a nearer neighbor without regard to the distance. In reality, an interaction boundary must exist, but it may be much greater than the sector radius $R C$, as detection of companions by vision, lateral line, and olfaction may be possible outside this range. Simulation in which the boundary distance was introduced and assumed to be at least $10 \mathrm{BL}$ in addition to parameters in the standard run resulted in no significant dif- 
ferences. It is considered that the boundary distance little affects the results of the simulations so long as a probable value is chosen for it.

The model presented here is obviously much simpler than the behavior of real fish. However, the study is sufficient to demonstrate the importance of parallel orientation movement as well as mutual attraction. The results of the simulation obtained here support the two-order system of schooling suggested by SHAw. ${ }^{8)}$

OKuno $^{17)}$ classified modes of life in marine fishes into four types by interlacing categories of movement and sociality. YAMAGISHI ${ }^{18)}$ also distributed fish populations based on two tendencies, attractive-repulsive and migratory-resident, and regarded parallel orientation as an extremely strong attraction. When we consider interactions between individuals underlying these modes of life, the significance of parallel orientation movement should be emphasized. For example, a mode of life allowing both aggregation and migration is realized by the existence of a behavioral mechanism leading to parallel orientation in the presence of mechanism allowing mutual attraction. As discussed however, the presence of both mechanisms is necessary for the formation and maintenance of schooling phenomenon. Neither mechanism alone is sufficient. Differences in the degree of parallel orientation may exist among various schooling species. The presence of this ability is an adaptation to their modes of life or their environments. For example, fish living in the ocean must keep on moving to get food and to reach a spawning site. As refuges and landmarks are rare in their environments, it must be companions that should be the most depended upon, and strong cohesion must be required to survive. Such species must have a highly developed abilities to allow the group to move cohesively.

The simulation method is limited in ability to clarify actual complex biological systems. However, results provide a useful guide in the conduct of further biological research.

The main objective of this paper was to simulate schooling. In the next phase of research, many sorts of modification will be made on the model. For example, simulations can be made to answer questions of whether or not a consistent leader appears when members of a school are heterogeneous in their mode of movement and of how a school behaves when only a part of the school reacts to an external stimulus. Recently, simula- tion of fish behavior to fishing gear have been made to investigate fishing tactics. ${ }^{12,19}$ In addition; if temporal and spatial scales are made greater and a fish school is regarded as a unit instead of individual, it may be possible to simulate fish migration by synthesis of oceanographic data and biological knowledge of reactions and orientations to environmental stimuli.

\section{Acknowledgements}

The author wishes to express his gratitude to Professor T. KurokI, Nihon University, for critically reviewing the manuscript.

\section{References}

1) B. L. Partridge and T. J. Pitcher: J. Comp. Physiol., 135, 315-325 (1980).

2) Y. Ono: Ann. Anim. Psychol., 2, 65-75 (1936).

3) H. Kinishita: in "Current Problems in Biological Science" (ed. by A. OKaJima and $\mathbf{K}$. MaruYama), Vol, 1, Iwanami Shoten, Tokyo, 1975, pp. 123-145.

4) M. Inoue: La Mer, 13, 79-90 (1975).

5) E. Shaw: Am. Mus. Novitates, No. 2446, 1-16 (1971).

6) J. C. van Olst and J. R. Hunter: J. Fish. Res. Bd. Can., 27, 1225-1238 (1970).

7) B. L. Partridge, T. Pitcher, J. M. Cullen, and J. WILson: Behav. Ecol. Sociobiol., 6, 277-288 (1980).

8) E. SHAW: in "Development and Evolution of Behavior" (ed. by L. R. ARonson et al.), W. H. Freeman, San Francisco, 1970, pp. 452-480.

9) I. Aok1: Bull. Ocean Res. Inst. Univ. Tokyo, No. 12, 1-65 (1980).

10) S. SAKAI and R. Suzuki: Rep. Research Group on Medical Electronics and Biological Engineering, No. MEB73-4, 1-12 (1973).

11) T. Inagaki, W. SaKamoto, and T. Kuroki: Bull. Japan. Soc. Sci. Fish., 42, 265-270 (1976).

12) K. Matuda and N. SannomiYa: Bull. Japan. Soc. Sci. Fish., 46, 689-697 (1980).

13) D. B. SinifF and C. R. Jessen: Advances in Ecological Researches, 6, 185-219 (1968).

14) F. J. Rohlf and D. Davenport: $J$. Theoret. Biol., 23, 400-424, (1969).

15) E. Yano: Res. Popul. Ecol., 20, 105-122 (1978).

16) R. A. J. TAYLOR: J. Anim. Ecol., 48, 577-602 (1979).

17) R. Okuno: Pub. Seto Mar. Biol. Lab. Kyoto Univ., 11, 281-336, (1963).

18) H. Yamagishi: Current Problems in Ecology, Kodansha, Tokyo, 1973, pp. 200-205.

19) T. Mura: Bull. Japan. Soc. Sci. Fish., 45, 17-23 (1979). 